\title{
REFLEXIONES SOBRE PSICOLOGİA DE LA COMUNICACIÓN COMO ÁREA ESPECIALIZADA
}

Reflections on the psychology of communivation as a specialized area

Amaro La Rosa*

\section{Resumen}

El artículo presenta los lineamientos básicos de la Psicología de la Comunicación como área especializada. Parte de criterios conceptuales, describe el desarrollo de la docencia e investigación a nivel internacional y formula algunas pautas para el trabajo teórico y aplicado.

Palabras clave: Psicología, comunicación, teoría.

\begin{abstract}
The article presents the basic features of Psychology of Communication as a specialized area of Psychology. Starting from conceptual criteria, it describes the development of research and teaching internationally and proposes some guidelines for theoretical and applied work.
\end{abstract}

Keywords: Psychology of Communication, theory.

\footnotetext{
* Psicologo Social y Comunicador. Docente de la Universidad Femenina del Sagrado Corazón. amaro@unife.edu.pe
} 


\section{PUNTO DE PARTIDA}

La presente propuesta parte de la experiencia práctica de varias décadas en la docencia e investigación en Psicología de la Comunicación. Nuestra formación fue en el terreno, recopilando datos, investigando, publicando y conversando con algunos colegas que enseñaban la materia así como recibiendo el aporte de nuestros estudiantes. Desde la década de los 90 contamos con Internet como recurso tecnológico que facilita el intercambio con especialistas de otras latitudes y nos permite estar al tanto de los últimos estudios e investigaciones sobre el particular, que se publican con tal profusión, que sería materialmente imposible revisar todo lo que aparece en la red. Podemos seleccionar el material que revisaremos en detalle, pero también asistir constantemente a teleconferencias, participar en seminarios virtuales, atender a webinars, escuchar podcasts de los más renombrados especialistas o revisar los materiales de curso de las universidades más renombradas del mundo. Todo ello nos permite ahora formular algunas ideas básicas que presentamos a la consideración de los lectores.

\section{CONSIDERACIONES TEÓRICAS SOBRE PSICOLOGÍA DE LA COMUNICACIÓN}

Nuestra área, paulatinamente ha ido construyendo conocimientos y cuenta con un objeto de estudio propio identificado a nivel internacional.

La división 46 de la American Psychological Association, fundada en 1987, está dedicada a la Psicología de los Medios (Media Psychology) y explícitamente señala en su sitio web que "se enfoca en los roles que el Psicólogo desempeña en diversos aspectos de los medios, incluyendo a la radio, la televisión, el cine, el video, los medios impresos, las revistas y las nuevas tecnologías; pero sin limitarse a ellos. Intenta promover lainvestigación sobreel impacto de los medios sobre el comportamiento humano, facilitar la interacción entre los psicólogos y los representantes de los medios, enriquecer la enseñanza, la capacitación y la práctica de la Psicología de la Comunicación. Igualmente, preparar a los psicólogos para interpretar la investigación psicológica para el público en general y para otros profesionales".
Para Fischoff (2005) la Psicología de los medios emplea las teorías, conceptos y métodos de la Psicología para estudiar el impacto de los medios masivos sobre los individuos, grupos y culturas. Considera que es muy dinámica y recíproca la naturaleza de los medios y sus consumidores. Resume su criterio especificando que la Psicología de la Comunicación está relacionada con los parámetros sociales y psicológicos de la comunicación entre las personas, la cual está mediada por alguna tecnología.

Consideramos que Wikipedia, la gran enciclopedia de Internet que cuenta al 17 de octubre de 2012 con 4,077,081 artículos en inglés, 927,686 artículos en español; y es publicada actualmente en 285 idiomas no es un recurso $100 \%$ válido desde el punto de vista académico. No obstante consignamos la entrada sobre Psicología de los Medios en tanto en nuestro caso al menos, alude a fuentes confiables, algunas de las cuales ya habíamos revisado previamente. Afirma que el estudio psicológico de los medios de comunicación ha surgido como disciplina académica y profesional debido a la demanda comercial y social para aplicar la teoría psicológica y la investigación al impacto de los medios y las nuevas tecnologías de la comunicación en nuestra sociedad y en los individuos, tanto en el ámbito académico como en el no académico.

Desde el punto de vista de Pamela Routledge, especialista estadounidense, la Psicología de la Comunicación es un campo muy dinámico que comprende al sistema de la comunicación en sus conjunto. Routledge señala que el campo de la Psicología de la Comunicación debe incluir el trabajo que se hace como el que necesita hacerse.

El trabajo que se hace efectivo en el área, según Routledge, es una suerte de bucle sin inicio ni final que incluye al desarrollo de la tecnología, la producción del contenido, la percepción del contenido y la respuesta del usuario. De modo similar al que Albert Bandura describe la teoría social cognitiva como la acción recíproca entre el medio ambiente, la conducta y la cognición, la Psicología de los medios evalúa el proceso interactivo del sistema, tal con se muestra en el gráfico 1. 
Naturalmente, esto no significa que el Psicólogo se dedique necesariamente a estos cuatro aspectos. En los países altamente desarrollados donde existe la súper especialización es posible que un Psicólogo pueda dedicarse a tiempo completo a ello. En países como el nuestro es probable que investigue la percepción del contenido y la respuesta del usuario, así como que se alterne esto con otras actividades. Sin embargo, cabe señalar la atingencia de que actualmente es posible desarrollar labores de asesoría en cuanto a producción de contenidos, pues existe una industria local emergente en este particular, y que crecerá aceleradamente a decir de las tendencias del mercado.

\section{Gráfico 1}

\section{Campo de la Psicología de los Medios según Routledge}

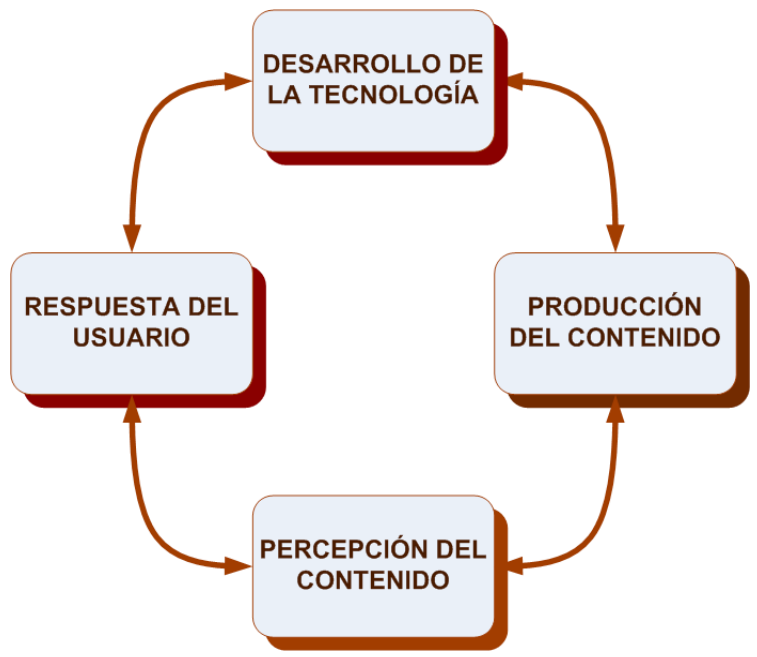

Elaboración: Routledge (s/d). Traducción: ALR

Whittemore (2008) entiende que se trata de un área interdisciplinaria especializada de la Psicología que de una manera simple puede decirse que está enfocada en la investigación erudita (académica) de los medios. Desde su perspectiva esta nueva área de la Psicología tiene sus raíces en una variedad de campos que incluyen la Sociología, la tecnología, los estudios sobre comunicación y medios masivos y la Psicología. Involucra tanto a investigadores como a profesionales especializados.

Wachelke y Segala (2005) partiendo de la concepción de la comunicación como proceso social básico, entienden que es un objeto de estudio interdisciplinario de la Psicología para el estudio de las intenciones del comunicador o del comportamiento del receptor.

Cuesta (2000) Moral e Igartua (2005) así como Pastor (2006), entre otros, prefieren denominarla Psicología social de la comunicación. Desde una perspectiva psicosocial conciben a la comunicación como vehículo de interacción social, confluyendo necesariamente en esta actividad los factores psicológicos y el entorno social.

Para Cabada (2004) la disciplina denominada Psicología de la Comunicación, que estudia los fundamentos y parámetros comunicativos de la conducta humana y animal, pero es demasiado genérica y su enfoque se realiza desde la Psicología individual y básica y no desde la Psicología Social.

Jackson, R. (2004) sostiene la existencia de tres criterios de aproximación a los medios:

- Contenido: Lo que presenta el medio.

- Exposición: la forma en que se percibe el mensaje.

- Efectos: El resultado que se deriva de la percepción de los mensajes masivos.

Estimamos que en todo caso estas tres aproximaciones, aun cuando tienen sus particularidades confluyen hacia el objetivo común de la comprensión de las modalidades de vinculación de los perceptores con los medios de comunicación

A decir de Giles (2003) ciertas áreas de la Psicología se han delimitado a partir de una temática específica y este es el caso de la Psicología de la Comunicación, que en términos reales, puede incorporar perspectivas de otras áreas sin ser dominada por ninguna perspectiva teórica ni enfoque metodológico en particular. Y aquí encuentra Giles un hecho significativo que vale la pena comentar: Muchos de los conocimientos obtenidos en este terreno provienen de los estudios de especialistas de otras disciplinas, algo similar con lo que sucede en general con el área de la Comunicación, en la cual 
un sinnúmero de conocimientos y enfoques teóricos significativos provienen del trabajo de especialistas en otras áreas del conocimiento.

Adoptando la perspectiva de Baumeister y Finkel (2010) puede pensarse que los psicólogos sociales a veces tienen como tarea la comprensión de las situaciones, lo que hasta cierto punto podría igualmente trasladarse a la percepción de lo que significa la Psicología de la Comunicación desde la visión de los no iniciados.

Por nuestra parte entendemos que la comunicación es una actividad humana que ha evolucionado a través de los tiempos desde las formas más primitivas de intercambio, donde probablemente existían muchas similitudes con la comunicación de los antropoides actuales, hasta las formas más avanzadas de representación simbólica de la realidad que involucran el empleo de recursos tecnológicos para el intercambio, que cada vez se vuelven más sofisticados. Desde luego que las modalidades de comunicación han sufrido profundas transformaciones, que se han acelerado especialmente durante las últimas décadas del siglo XX y lo que va del XXI.

A pesar de ser relativamente joven, el estudio científico de la Comunicación ha experimentado un incesante desarrollo en los últimos años, como resultado de la investigación y de la construcción teórica, tareas en las cuales han brindado sustanciales contribuciones investigadores formados, en especial, en el área de las Ciencias Sociales y en nuestro caso creemos que no debe desconocerse el aporte de la Psicología.

Uno de los requisitos fundamentales para que cierta disciplina pueda catalogarse como científica, es contar con un objeto de estudio propio; vale decir, que se encargue de analizar determinada clase de fenómenos producidos en la realidad.

La Psicología de la Comunicación no se exime de la dificultad que existe para delimitar el área de estudios de la Comunicación. Según Martino (2003), ello se debe a su multidimensionalidad, a la diversidad de enfoques así como a que se mantiene en competencia con otras disciplinas sociales, por el análisis de una serie de aspectos de la realidad.
Ciertamente no resulta tarea sencilla precisar cuáles son los fenómenos que comprende la Comunicación, pues hasta donde recordamos, los criterios pueden tener mayor o menor extensión, de acuerdo al paradigma y el enfoque teórico de los autores. En cualquier caso, y a riesgo de parecer demasiado comprensivos, podríamos decir que en ella están involucrados aquellos procesos de intercambio de productos o elementos simbólicos. No obstante aún este criterio podría involucrar los procesos comunicacionales que se dan en el marco de la entrevista y la psicoterapia (Psicología Clínica), el análisis de los fenómenos comunicacionales que se evidencian al interior de una organización (Psicología Organizacional) o igualmente a lo implicado en proceso de enseñanza-aprendizaje que a decir de Paulo Freire y Mario Kaplún, entre otros, supone necesariamente comunicación. Y no es que queramos exagerar la importancia de la comunicación sino nuestra intención es buscar la comprensión y buscar el deslinde de lo que implica nuestra área de conocimiento.

Otro de los aspectos problemáticos es que se recogen los planteamientos de distintos enfoques teóricos de la Psicología. Personalmente siempre hemos discrepado con los dogmatismos o los purismos, y estamos convencidos que para criticar algo primero debemos conocerlo cabalmente, remitiéndonos en la medida de lo posible a la propia fuente donde se encuentran los planteamientos del autor. Ahora bien, la crítica supone el acuerdo o desacuerdo con los planteamientos; pero en ningún caso la descalificación, puesto que cada enfoque tiene sus propios méritos los cuales deben reconocerse así como sus vacíos o aspectos cuestionables, que no tienen porqué desconocerse. Para decirlo en términos del análisis FODA: cada teoría tiene sus fortalezas y oportunidades, pero también sus amenazas y debilidades, que es menester conocer cabalmente, para no convertirnos en criticastros.

Compartimos con las Ciencias Sociales, el problema que destaca Vizer (2002), cuando expresa que el conjunto de formaciones conceptuales que implica la Comunicación, muestra la tendencia a interpretar y reinterpretar de manera permanente su objeto de estudio (la comunicación). Esto resulta explicable dada la naturaleza específica de los fenómenos que investiga. En el caso de la comunicación masiva, es evidente que no representa una realidad permanente, 
estática, sino que más bien de manera constante aparecen nuevos asuntos y manifestaciones, los cuales merecen la atención de los especialistas determinando reformulaciones del campo, Esto es lo que ha ocurrido por ejemplo cuando el desarrollo de las nuevas tecnologías determinó a su vez el desarrollo de otras modalidades de comunicación que era necesario abordar,

En nuestro caso queremos plantear la necesidad de una visión más comprensiva del objeto de estudio de nuestra disciplina y una reformulación que permita elaborar nuevos modelos, que hagan posible la descripción y explicación de las nuevas modalidades de comunicación tales como la CMC (comunicación mediada por computadoras) y de su impacto en el comportamiento social de los seres humanos.

La ciencia supone la reconstrucción de la realidad y tratándose de los fenómenos psicológicos y de la comunicación que son la resultante de múltiples variables, porello, cabe visualizarla desde la perspectiva conjunta de disciplinas diversas para entender en sus reales dimensiones una problemática bastante compleja. Entendemos por ello que las características de nuestro objeto de estudio requieren necesariamente un análisis interdisciplinario.

En este sentido, coincidiendo con Anolli (2010) estamos convencidos que Psicología de la Comunicación es un área interdisciplinaria en la cual confluyen los conocimientos tanto de la Psicología como de la Comunicación, para la comprensión de los procesos comunicacionales, tal como intentamos expresar en el gráfico 2. En cierto modo repite la tendencia mostrada muchas décadas atrás cuando surgiera la Psicología Social, como fruto del interés por el comportamiento social tanto de Psicólogos como de Sociólogos.

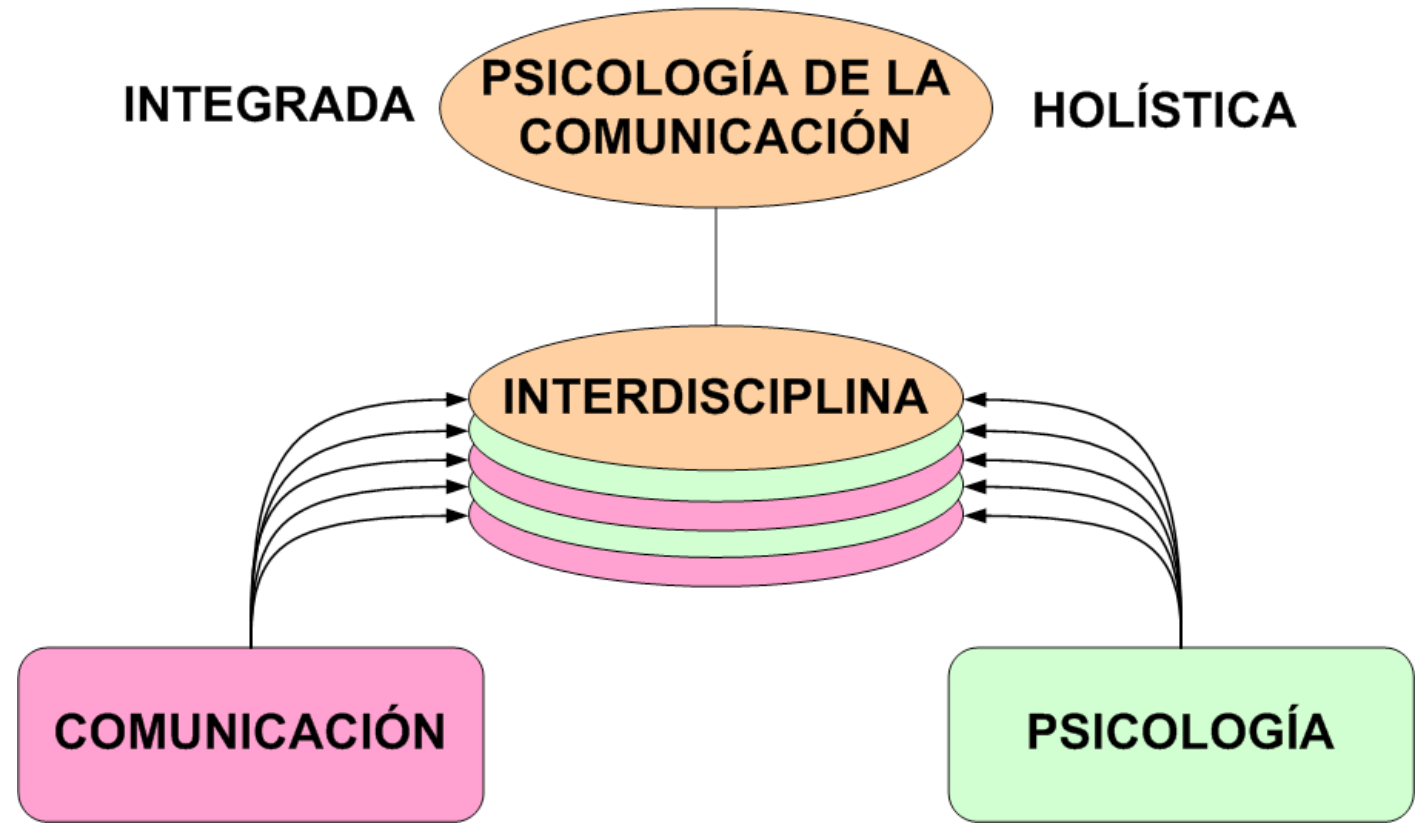

Elaboración: ALR 
Nuestro punto de vista, manifestado en varios de nuestros trabajos (La Rosa, 1999, 2001 y 2002), es concordante con la tendencia actual hacia la convergencia en las Ciencias Sociales. Si bien cada ciencia tiene su propio objeto de estudio, se estima ahora que es necesario estrechar los puntos ce coincidencia entre ellas con miras a la comprensión más clara de la realidad. Esto por cierto no se descubre meramente ahora pues recordamos haber leído a Jean Piaget planteando un punto de vista concordante con esta convergencia, que en la práctica permite un conocimiento holístico de la realidad.

En la ciencia, los paradigmas orientan el quehacer investigativo, desde la observación de la realidad hasta la interpretación de los resultados obtenidos. Para Derrida cada paradigma puede visualizarse como una posición teórica, que involucra un cierto campo de visión con un horizonte limitado. De acuerdo al paradigma orientador de su percepción del mundo, el especialista verá la realidad desde su propia mirada científica, que ha desarrollado como consecuencia de su praxis profesional. Para decirlo en otros términos, el científico hará su observación de los fenómenos, los cuales posteriormente describirá y explicará, en consonancia con su criterio de lo que significa la realidad. Naturalmente, la validez y la confiabilidad de los resultados obtenidos estarán en consonancia con el respeto a los principios de la investigación científica.

Ciertamente la Psicología de la Comunicación hace uso de un conjunto de métodos y técnicas de investigación científica para conseguir la meta de acercarse al conocimiento de su objeto de estudio: $\mathrm{La}$ comunicación.

De la apreciación de la unidad del conocimiento, que parte de Piaget, se desprenden a nuestro entender las orientaciones metodológicas para la investigación de la comunicación, la cual debería emplear de manera integrada métodos, técnicas y criterios conceptuales procedentes de diversas áreas del conocimiento. Desde la perspectiva de Thompson (1998) así como de Defila y Di Giulio (1999), la idea es contribuir al logro de conocimientos que trasciendan las actuales fronteras disciplinarias.
Entendemos que la investigación interdisciplinaria en Psicología de la Comunicación nos plantea una serie de demandas:

- Una visión más amplia de los investigadores para salir de los marcos de su disciplina y aceptar que el contexto de referencia para el conocimiento de la realidad posee mayor amplitud que su esquema perceptual previamente formulado e interiorizado, a partir de sus paradigmas con que cuenta.

- La formación de equipos de trabajo donde las premisas sean el trabajo en equipo y la visión holística de la problemática. Esto obliga por cierto a superar visiones individualistas, intentando la elaboración de un pensamiento colectivo, donde cada uno trabaje activamente por el conjunto.

- La capacitación y reciclaje de los investigadores para que estén en condiciones de asumir una visión interdisciplinaria desde el propio planteamiento del problema.

- Contar con la suficiente flexibilidad para incorporar nuevas connotaciones y criterios conceptuales con los cuales no ha estado familiarizado, pero que resultan indispensables para comprender el objeto de conocimiento.

- Guardar coherencia epistemológica durante la investigación. Ciertamente el acento interdisciplinario no está dado en el enfoque de alguna de las fases, pues como Díaz (1999) estamos convencidos que cualquier proceso de investigación científica involucra la toma de decisiones en todas y cada una de sus etapas, desde la percepción del objeto de estudio, pasando por el entramado conceptual, el planteamiento de la propuesta metodológica y el análisis de los resultados, los cuales no solamente deben guardar coherencia formal sino también epistemológica.

- Es necesario del mismo modo innovar en lo referido a la evaluación. La calidad y relevancia de los trabajos interdisciplinarios no pueden analizarse basándose en los mismos parámetros de la investigación disciplinaria tradicional (Spaapen, Wamelink y Dijstelbloem, 2003)

Estamos convencidos que puede responderse a estas expectativas, con estudios serios que 
analicen nuestra problemática, pues los especialistas latinoamericanos han proporcionado aportes significativos en la investigación de la comunicación, los cuales son reconocidos a nivel mundial.

\section{DOCENCIA E INVESTIGACIÓN PSICOLOGÍA DE LA COMUNICACIÓN} EN

El área ha sido reconocida por la American Psychological Association (División 46), y comprende:

- Diagnóstico.

- Investigación.

- Evaluación.

- Modificación de conducta.

- Desarrollo de la personalidad y de las competencias del individuo.

A nivel mundial, la Fielding University ubicada en California ha sido la pionera en ofrece un programa acreditado de $\mathrm{Ph}$. D en Media Psychology. Sin embargo, las oportunidades educacionales en el campo están creciendo. Desde 2007 la California State University tiene un programa de Master en Medios con base psicológica y hay programas similares orientados hacia la comunicación en University of Chicago, University of Denver, University of Iowa, New York University, Stanford University y el reconocido Massachusetts Institute of Technology. La Universitat Autónoma de Barcelona dicta un Máster en Psicología de la Cognición y Comunicación Igualmente, resulta materia imprescindible en las carreras de Comunicación. Por su parte la Massachusets School of Professional Psychology ofrece el Programa de Maestría en Psicología de los medios.

Actualmente se enseña Psicología de la Comunicación en un sinnúmero de centros de estudio a nivel mundial y es materia obligatoria en las carreras de Comunicación.

Se editan igualmente publicaciones especializadas tales como Journal of Media Psychology y Media Psychology Review
El primer Laboratorio de Psicología de los Medios abrió sus puertas en enero de 1996 en la Califormia State University de Los Angeles, bajo la supervisón del Dr. Stuart Fischoff, luego Editor principal de Journal of Media Psychology y luego Presidente fundador de la División 46 de la APA en 1986. En 1990, el Dr Fischoff había dictado el primer curso de Psicología de los Medios en la CSLA

Existen asimismo centros de investigación altamente especializados en temáticas específicas. Tal es el caso del Instituto para la Investigación de los medios y la Comunicación de la Universidad Técnica de Ilmenau. El instituto afirma que la Psicología de los medios es una de las subáreas aplicadas de la Psicología que trata la conducta humana y los procesos mentales relacionados con los medios de comunicación. Agrega que con cada innovación surgen nuevas interrogantes que debe responder la investigación.

Las temáticas que trabaja actualmente el Instituto son:

- Psicología de la Comunicación Online.

- Psicología de la Comunicación Móvil.

- Psicología de la Comunicación Humano-Robot.

- Psicología de la Realidad Virtual.

El Human Communication Laboratory (COMM: LAB) de la Columbia University estudia la conducta comunicativa desde una perspectiva psicosocial.

El CESCOM (Centro degli Studi per le Scienze della Comunicazione) funciona en el Dipartimento di Scienze Umane de la Università degli Studi di Milano Bicocca, desarrollando investigaciones en las siguientes áreas:

- Comunicación humana (modelos y métodos).

- Metodología y técnicas de investigación en interacción comunicativa (codificación y análisis de conducta verbal y no verbal, tanto cara a cara co o mediada por computadoras).

- Comunicaciòn no verbal ( expression del rostro, gesto, mirada, tactil, proxémica y cronemática)

1. http://www.tu-ilmenau.de/fakmn/1-Media-Psychology.4830.0.html?\&L=1 
- Comunicación intercultural y entrenamiento multicultural de la mente

- Juegos serios y simulaciones interactivas para el entrenamiento de competencias finas.

- Emoción(expresiones emocionales y regulación de emociones

- Nuevas perspectivas en comunicación errada (decepción, seducción e ironía).

- Comunicación afectiva y sistemas e-learning

- Psicología Positiva (optimismo y resiliencia)

- Sentido de presencia en medioambientes mediados.

- Realidad virtual para entrenamiento y terapia.

El Media Psychology Program que forma parte del Research Center for Group Dynamics de la University of Michigan se propone comprender la influencia de los medios masivos en la conducta individual y grupal así como en las mentes individuales. Su investigación se desarrolla en dos sub áreas:

- Salud y los medios

- Violencia en los medios y conducta agresiva.

El Medienpsychologischen Labor der Universität Siegen, entiende que la especialidad tiene desarrolla los siguientes temas de investigación:

- Investigación básica y desarrollos teóricos en Psicologìa de los medios.

- Uso de los medios y su efecto para los clientes en la industria de los medios.

- Desarrollo de nuevas y conceptualmente apropiadas escalas de medición para diversos propósitos.

- Concepción y evaluación de cursos de entrenamiento en competencia de medios/ medios digitales para docentes

- Concepción y evaluación de cursos de comunicación para la salud.

- Concepción y evaluación de programas de servicio en el terreno de la salud.

The Media Psychology Research Center (MPRC) es una organización din fines de lucro que funciona en Estados Unidos, dedicada a la investigación de los medios, la evaluación y educación. Su investigación trata

- La manera en que el público consume produce y distribuye información utilizando tecnologías de medios.

- El impacto de los medios en la sociedad y en los individuos.

- La interacción de los medios con la experiencia cotidiana.

- El uso positivo de los medios

The Media Effects Research Laboratory de la Pennsylvania State University investiga en dos lineas:

- Medios tradicionales: Efectos de la televisión, filmes de entretenimiento y videojuegos.

- Nuevos medios: Efectos de los medios online y de la tecnología de las telecomunicaciones

En Rusia el MIND Lab de la Moscow City University of Psychology and Education se especializa en:

- Psicología de la comunicación mediada por Internet

- Entretenimiento y cognición

- Estudios de género en Internet

\section{NUESTRO CAMPO DE TRABAJO}

A nuestro entender la Psicología de la Comunicación debería reconocerse como una de las áreas de desempeño profesional del Psicólogo, involucrando las siguientes líneas de trabajo:

- Investigación: Analiza los fenómenos de comunicación que se dan en la producción de los mensajes masivos, en la recepción de los mismos y los efectos que pueden derivarse de los mensajes y la exposición a los productos mediáticos y recursos tecnológicos. Realiza estudios para la construcción de conocimientos y enfoques teóricos. Desarrolla investigación aplicada en su área apelando tanto a métodos cuantitativos como cualitativos y maneja toda la gama de recursos metodológicos que se emplean para estudiar los mensajes masivos y la audiencia. 
Esto supone el análisis de los efectos de los medios en general así como del impacto de los mensajes publicitarios o propagandísticos. Tal como intenta expresarlo el gráfico 2 esto supone estar al tanto de las nuevas demandas de la sociedad como de las nuevas problemáticas que van surgiendo en el ámbito de la comunicación.

\section{Gráfico 3}

\section{Psicologia de la Comunicación, Investigación y Realidad}

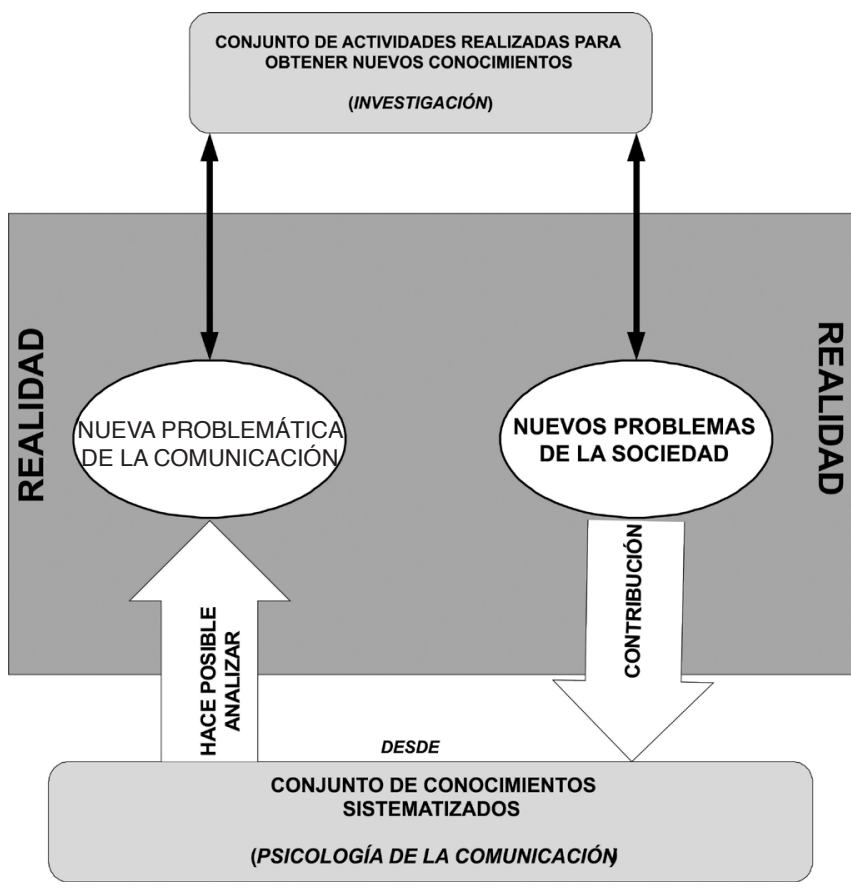

Elaboración: ALR

- Consultoría: Provee asesoría y orientación especializada para la producción de mensajes y su análisis por parte de públicos diversos. Igualmente brinda servicios de consultoría en cuanto a la lectura crítica de mensajes. Interviene asimismo en procesos de modificación de conducta empleando medios masivos. Brinda asesoramiento psicológico en diseño y ejecución de campañas políticas.

-Docencia: Produce contenidos para el aprendizaje del lenguaje de los medios y de la propia construcción de los discursos mediáticos. Aplica herramientas para el desarrollo de competencias comunicativas.
- Difusión del conocimiento científico: Desarrolla y produce instrumentos y recursos para difundir los conocimientos sobre el comportamiento y los resultados de la investigación en Psicología.

Creemos que se trata de un área sumamente importante para el trabajo del Psicólogo. Sin embargo existe un número pequeño de especialistas locales, quienes desde sus propias perspectivas teóricas y metodológicas trabajan en universidades y centros de investigación. En nuestro medio me permitiría citar por su trabajo constante y sus publicaciones a Víctor Montero de la Universidad Nacional Mayor de San Marcos, Sandro Macassi de la Pontificia Universidad Católica y a Julio Hevia de la Universidad de Lima. En la práctica somos un pequeño número de especialistas que sin lugar a dudas tenemos un acercamiento bastante marcado con la Comunicación, pero que somos la minoría en cuanto a participación en las actividades profesionales de la Psicología. Lo mismo ocurre por cierto a nivel internacional, pues como de la Universidad de Puerto Rico nos comentaba, el número de presentaciones del área en Congresos y otros certámenes de Psicología es muy limitado. Sin que abjuremos de nuestra formación profesional como Psicólogos, sino que por la propia naturaleza de nuestro trabajo, encontramos un mayor eco entre estudiantes y profesionales de Comunicación

Entendemos que probablemente en nuestro medio sea muy limitada la demanda de este tipo de profesional y por ende el interés de estudiantes y profesionales por ella. Sin embargo esto no desmerece la importancia de la especialidad.

\section{REFERENCIAS}

Anolli (2010) Prima lezione di Psicologia della comunicazione. Roma-Bari: Editori Laterza

APA (s/d) Welcome to Division 46 of the American Psychological Association: Media Psychology. http://www.apa.org/divisons/div46

Baumeister, R. y Finkel, R. (2010) Advanced Social Psychology: The state of the science New York: Oxford University Press 
Cabada, M. (2004) Análisis psicosocial de la comunicación audiovisual de masas actual Tesis de Doctorado en Psicología Madrid: Universidad Complutense de Madrid

Cuesta, U (2000) Psicología social de la comunicación. Madrid: Cátedra

Defila, R. y Di Giulio, A. (1999) "Evaluation criteria for Inter and Transdisciplinary Research", en Panorama Special Issue, 1, 1999, pp. 5-11. Consulta 25 de noviembre 2010. Disponible en Internet. http://www.ikaoe.unibe.ch/forschung/ip. Specialissue.Pano.1.99.pdf

Díaz, N. (1999) Apuntes para empezar a pensar una reconstrucción teórico-metodológica latinoamericana. Ponencia en el Seminario Internacional Tendencias y retos de la investigación en comunicación en América Latina, Lima.

Fischoff, S. (2005) Media Psychology: A Personal Essay in Definition and Purview. Online paper http://www.apa.org/divisions/div46/images/ MEDIADEF.pdf

Giles, D. (2003) Media Psychology Mahwah, New Jersey: Lawrence Erlbaum Associates Publishers

Jackson, R. (2004) A cognitive psychology of Mass Communications Mahwah, New Jersey: Lawrence Erlbaum Associates Publishers

Moral, F. e Igartua, J. (2005) Psicologia social de la comunicación: aspectos teóricos y prácticos. Archidona: Aljibe

La Rosa, A. (2002) Psicología de la Comunicación: De los mensajes a los efectos Lima: Escuela de Periodismo Jaime Bausate y Mesa

La Rosa, A. (2001) "Investigación de la comunicación y autopistas de la información", en Comunifé, 1, (1), pp. 66-80

La Rosa, A. (1999) Los desafíos epistemológicos de la investigación de la comunicación en la era del ciberespacio Ponencia presentada en el Seminario Internacional "Tendencias y retos de la investigación en comunicación en América Latina” Lima, julio

Fecha de recepción: 19 de octubre, 2012

Fecha de aceptación: 10 de noviembre, 2012
Martino, L. (2003) "Épistemologie de la communicattion: Scepticisme et intelligibilite du savoir communicationnel", Les Enjeux de l'information et de la communication, 29 septembre. Disponible en Internet: http://www.ugrenoble3.fr/les_enjeux/2003/Martino/index.html

Murphy, S.M. (2007). A social meaning framework for research on participation in social online games. Journal of Media Psychology, 12. Retrieved October 15, 2007 http://www.calstatela.edu/ faculty/sfischo/A_Social_Meaning_Framework_ for_Online_Games.html

Pastor, Y (2006) Psicología social de la comunicación: aspectos básicos y aplicados Madrid: Pirámide.

Routledge, P. (s/d) What is Media Psychology? The Media Psychology Research Center. Online paper, accesado 20 de Julio de 2010 http://mprcenter. org/?page_id=16

Spaapen, J., Wamelink, F. y Dijstelbloem, H. (2003) "Towards the evaluation of transdisciplinary research" en Landscape Studies: Potential and Limitations, DELTA SERIES, Wageningen, 2003, pp. 148 -157. Disponible en Internet: http://www. wur.nt/delta/series $2 . h t m l$

Thompson, J. (1998) Notes Toward a Social Epistemology of Transdisciplinarity, en Rencontres Trandisciplinaires. Bulletin interactif du Centre International de Recherches et Études transdisciplinaires (CIRET) $\mathrm{N}^{\circ} 12$ février. Disponible en Internet: http://perso.club-internet. $\mathrm{fr} / \mathrm{nicol} / \mathrm{ciret} / \mathrm{bulletin} / \mathrm{b} 12 \mathrm{c} 2 . \mathrm{html}$

Vizer, E. (2002) La trama invisible de la vida social: comunicación, sentido y realidad. Ponencia presentada en el VI Congreso Latinoamericano de Investigadores de la Comunicación, Santa Cruz de la Sierra, Junio.

Wachelke, J. y Segala. M. (2005) Interdisciplinaridade em Psicologia Social e Comunicaçao Social. Ponencia presentada en el XXVIII Congreso Brasileiro de Ciências da Comunicaçáo, Rio de Janeiro, Setiembre. 